\title{
Bauhinia forficata L. e sua a ação hipoglicemiante
} Bauhinia forficata $L$. and its hypoglycemic action

\author{
Bauhinia forficata L. y su acción hipoglicémica \\ Maria Alana Neres de PONTES ${ }^{1}$ \\ Dijaci Santos de LIMA ${ }^{2}$ \\ Heloísa Mara Batista Fernandes de OLIVEIRA ${ }^{3}$ \\ Abrahão Alves de OLIVEIRA FILHO ${ }^{4}$
}

${ }^{1}$ Graduanda em Bacharelado em Farmácia, Centro de Educação e Saúde,

Universidade Federal de Campina Grande - UFCG, 58175-000 Cuité-PB, Brasil

${ }^{2}$ Graduando em Bacharelado em Farmácia, Centro de Educação e Saúde,

Universidade Federal de Campina Grande - UFCG, 58175-000 Cuité- PB, Brasil

${ }^{3}$ Farmacêutica - Bioquímica - Doutora em Farmacologia,

Hospital Universitário Ana Bezerra - HUAB/EBSERH, 59200-000 Santa Cruz- RN, Brasil

${ }^{4}$ Farmacêutico - Bioquímico - Doutor em Farmacologia - Professor da Unidade Acadêmica de Ciências Biológicas - UACB,

Centro de Saúde e Tecnologia Rural, Universidade Federal de Campina Grande-UFCG, 58700-970 Patos- PB, Brasil

\section{Resumo}

A B. forfica $L$ uma das espécies mais populares do gênero Bahuina, pertencente à família Fabaceae, possui aproximadamente 300 espécies distribuídas principalmente pelas regiões tropicais do globo terreste. A pata-de-vaca nome popular dado a B. forficata tem suas diversas partes amplamente utilizadas para a preparação de produtos medicinais "caseiros" na terapia de diversas doenças, desde infecções, dores até como um agente hipoglicemiante para o tratamento da Diabetes. Os primeiros estudos clínicos com a pata-devaca se iniciaram no período de 1929 justificando o seu uso como hipoglicemiante e posteriormente estudos decorrentes no ano de 2004 validam o primeiro estudo clínico realizado, trazendo cada vez mais o interesse da comunidade cientifica para esta espécie medicinal. O propósito deste trabalho foi investigar e apresentar as propriedades farmacológicas, em especial hipoglicemiante, da $B$. forficata através de uma revisão narrativa da literatura. Metodologia: Foi realizada uma revisão narrativa da literatura científica, definindo-se como uma análise mais ampla dos conhecimentos já produzidos, tomando como base de dados trabalhos científicos na faixa anual de 2007 a 2017. Diante da revisão da literatura realizada foi possível evidenciar os efeitos hipoglicemiantes do extrato da B. forficata, bem como o seu emprego em diferentes formas farmacêuticas, desde pós a granulados. Concluiu-se que embora a $B$. forficata se mostre promissora para a área farmacêutica são necessários mais estudos associados a possíveis formas farmacêuticas que podem ser utilizadas, uma vez que o emprego de plantas medicinais na terapia de controle da diabetes seja uma forma de facilitar a adesão dos pacientes ao tratamento.

Descritores: Diabetes Mellitus; Hipoglicemiantes; Fitoterapia; Bauhinia.

\section{Abstract}

The B. forfica $L$ one of the most popular species of the Bahuina genus, belonging to the Fabaceae family, has approximately 300 species distributed mainly in tropical regions of the earth globe. The popular name paw given to B. forficata has its various parts widely used for the preparation of "home" medicinal products in the therapy of various diseases, from infections, pains to as a hypoglycemic agent for the treatment of Diabetes. The first clinical studies with the paw-of-cow began in the period of 1929 justifying its use as hypoglycemic and later studies in 2004 validate the first clinical study carried out, bringing increasingly the interest of the scientific community for this species medicinal. The aim of this paper was to investigate and present the pharmacological properties, especially hypoglycemic, of B. forficata through a narrative review of the literature. A narrative review of the scientific literature was carried out, defining itself as a broader analysis of the knowledge already produced, based on scientific studies in the annual range from 2007 to 2017. In the face of the literature revision carried out, the hypoglycaemic effects of the $B$. forficata extract, as well as its use in different pharmaceutical forms, from powders to granulate. It is concluded that although $B$. forficata appears to be promising for the pharmaceutical area, further studies are needed in relation to possible pharmaceutical forms that may be used, since the use of medicinal plants in diabetes control therapy is a way to facilitate adherence of patients to treatment.

Descriptors: Diabetes Mellitus; Hypoglycemic Agents; Phytotherapy ; Bauhinia.

\section{Resumen}

La B. forfica $L$ una de las especies más populares del género Bahuina, perteneciente a la familia Fabaceae, posee aproximadamente 300 especies distribuidas principalmente por las regiones tropicales del globo terreste. La pata de vaca nombre popular dado a $B$. forficata tiene sus diversas partes ampliamente utilizadas para la preparación de productos medicinales "caseros" en la terapia de diversas enfermedades, desde infecciones, dolores hasta como un agente hipoglicémiante para el tratamiento de la diabetes. Los primeros estudios clínicos con la pata de vaca se iniciaron en el período de 1929 justificando su uso como hipoglicemiante y posteriormente estudios resultantes en el año 2004 validan el primer estudio clínico realizado, trayendo cada vez más el interés de la comunidad científica para esta especie médico. El objetivo del presente trabajo fue investigar y presentar las propiedades farmacológicas, en particular hipoglicemiante, de la B. forficata a través de una revisión narrativa de la literatura. Se realizó una revisión narrativa de la literatura científica, definiéndose como un análisis más amplio de los conocimientos ya producidos, tomando como base de datos trabajos científicos en la franja anual de 2007 a 2017 . En vista de la revisión de la literatura llevada a cabo fue posible evidenciar los efectos hipoglucemiantes del extracto de B. forficata, así como su empleo en diferentes formas farmacéuticas, desde post a granulados. Aunque la B. forficata se muestra prometedora para el área farmacéutica, son necesarios más estudios asociados a posibles formas farmacéuticas que pueden ser utilizadas, ya que el empleo de plantas medicinales en la terapia de control de la diabetes es una forma de facilitar la adhesión de los pacientes al tratamiento.

Descriptores: Diabetes Mellitus; Hipoglucemiantes; Fitoterapia; Bauhinia.

\section{INTRODUÇÃO}

A diabetes mellitus é resultado de uma série de disfunções relacionadas à ação e/ou secreção da insulina. Essas disfunções podem envolver desde a destruição das células beta do pâncreas e resistência à ação aos distúrbios da secreção da insulina, entre outros. Todos estes mecanismos resultam no acúmulo da glicose no sangue, o que desencadeia o quadro característico da doença, a hiperglicemia, além de afetar o funcionamento dos principais órgãos, tais como: rins, cérebro, coração. Nesse contexto, destaca-se o uso de hipoglicemiantes que são agentes terapêuticos capazes de manter as concentrações plasmáticas de glicose dentro dos limites normais durante o maior tempo possível ${ }^{1}$. 
Um dos principais problemas relacionados ao controle da diabetes mellitus consiste na adaptabilidade dos pacientes ao tratamento, uma vez que se faz necessário um grande número de mudanças no comportamento destes, que vão desde o uso contínuo de medicamentos, alimentação restrita, realização frequente de exames, desta maneira acaba por influenciar negativamente na qualidade de vida dos pacientes e na evolução do quadro clinico ${ }^{2}$. Nesta perspectiva se faz necessária a utilização de terapêuticas que sejam de fácil aceitabilidade, tanto para o paciente como para os sistemas de saúde. Por conseguinte, as plantas medicinais entram como uma alternativa viável para resolução desta problemática, já que são amplamente aceitas por grande parte da população e poderiam facilmente diminuir os gastos com o tratamento ${ }^{3}$.

Desde os primórdios, as antigas civilizações buscam enfatizar a utilização das plantas para o tratamento e prevenção de enfermidades. Com o passar do tempo o conhecimento popular acerca do uso de plantas medicinais tem evoluído através da associação com os estudos técnico científicos voltados para a área da saúde, possibilitando então o surgimento de terapias alternativas ou até mesmo inéditas, seja pelo uso da droga vegetal ou de seus constituintes isolados ${ }^{4}$.

Diante do exposto, é importante ressaltar alguns estudos clínicos realizados entre os anos de 1929 e 2004 com espécies do gênero Bauhina, pertencente à família Fabaceae, que apresentam grande interesse da comunidade científica por apresentarem efeito hipoglicemiante. Dentre as espécies do gênero Bauhina ganha-se destaque a Bauhina forficata $L$ conhecida popularmente como "pata-de-vaca", nome este dado devido a aparência de suas folhas que são ricas no composto kaempfeitrina (possível marcador químico), encontrada e distribuída entre as regiões tropicais do globo. A pata-de-vaca tem sido amplamente utilizada na preparação de produtos medicinais "caseiros", com propriedades antidiabéticas, diuréticas, hipocolesteremiantes ${ }^{5-9}$

\section{MATERIAL E MÉTODO}

O presente trabalho trata-se de uma revisão narrativa da literatura científica, definindo-se como uma análise mais ampla dos conhecimentos já produzidos, a qual o estudo apresenta como finalidade integrar os conhecimentos já existentes sobre os aspectos das propriedades farmacológicas da Bauhinia forficata. Dessa forma, a revisão incluiu uma análise de pesquisas relevantes que oferecem um suporte, ou uma alternativa para a tomada de decisão e a melhoria da prática clínica ${ }^{10}$.

O material utilizado para a realização do estudo foi constituído de livros, artigos, monografias em português e inglês, que atenderam aos requisitos do tema abordado, apresentando informações confiáveis e publicados em bases de dados seguros. Foram priorizados os trabalhos com uma faixa anual de 2007 a 2017, com exceção de artigos clássicos que se apresentaram imprescindíveis ao presente estudo. Foram excluídos trabalhos que não apresentassem referências confiáveis, não abordassem a temática proposta, ou não atendesse aos critérios de inclusão.

Para a busca dos artigos, foram utilizados os seguintes descritores isolados ou em combinação: aspectos botânicos da planta Bauhinia forficata, constituintes químicos da planta, atividades farmacológicas da espécie, atividade hipoglicemiante, pata-de-vaca, fitoterapia. As bases de dados utilizadas foram: Americana e do Caribe em Ciências da Saúde (LILACS), biblioteca eletrônica
Scientific Electronic Library Online (SciELO), MEDLINE Medical Literature Analysis and Retrieval Sistem Online e BVS - Biblioteca Virtual em Saúde.

\section{RESULTADOS E DISCUSSÃO}

- Aspectos botânicos da planta

A variedade de espécies do gênero Bauhinia são conhecidas popularmente como "pata-de-vaca", "unha-dedente", entre outros ditos populares, sendo de grande utilidade na medicina. $\mathrm{O}$ gênero Bauhinia está incluído à família Leguminosae que apresenta cerca de 300 espécies distribuídas em áreas tropicais pelo o mundo ${ }^{11}$.

Essa espécie vegetal é de origem nativa da América do Sul, encontrada em países como Argentina, Paraguai, Uruguai, Bolívia e Brasil, onde no Brasil ela se estende principalmente nas regiões do Rio de Janeiro até o Rio Grande do Sul. Estudos relatam a presença de outras subespécies, como a B. forficata pruinosa (Vogel) Fortunato \& Wunderlin (sinonímia B. candicans Benth), e a B. forficata forficata ${ }^{12}$.

As folhas da leguminosa $B$. forficata apresenta uma semelhança gigantesca com outras folhas de outras espécies do gênero Bauhinia, porém, a presença de espinhos e suas flores com coloração exclusivamente branca são sua principal característica morfológica, assim facilitando na sua diferenciação ${ }^{13}$.

Dessa forma, a pata-de-vaca é considerada uma planta heliófila, ou seja, que necessita da exposição solar, sem preferência quanto às condições de umidade do solo, além de possuir características de exclusividade da mata semidecídua de altitude, ocorrendo com periocidade elevada, mas, com uma distribuição bastante irregular e descontínua $^{14}$.

A B. forficata apresenta-se como uma planta arbustiva, decídua, de tamanho elevado ou arbórea, perene, podendo atingir até $8 \mathrm{~m}$ de altura. Seus ramos são frágeis, pendulares, pubescentes ou glabros, com presença de acúleos gêmeos na axila foliar. Entretanto, suas folhas são alternas, ovais ou lanceoladas, divididas acima do meio, sendo composta por dois folíolos que se unem pela base, pouco afastadas, obtusas ou um pouco agudas, ou pode se encontrar acuminadas na base, com um formato arredondado ou subcodiformes, membranáceas, com característica de 9 nervos, e podendo medir de 8 a $9 \mathrm{~cm}$ de comprimento ${ }^{13}$.

Mesmo englobando uma variedade de estudos, esta espécie vegetal possui particularidades que não podem deixar de ser notadas e especificadas, como sua característica fenotípica, que se diferencia de região para região, bem como, as alterações que a mesma sofre com relação a sazonalidade, diferenciando espécies, e até tornando na maioria dos casos algumas produtoras de metabólitos secundários de importância, enquanto outras não produzem, ou seja, as "falsa" pata-de-vaca"

\section{- Constituintes químicos já identificados da planta (Fitoquímica da planta)}

A grande versatilidade da $B$. forficata em relação ao seu uso medicinal está relacionada a ampla variedade dos seus constituintes químicos que se encontram distribuídos de forma variável nos diferentes órgãos da planta e sofrendo também influência direta sobre: clima, localização geográfica dentre outros fatores influenciadores ${ }^{15}$.

Dentre os diversos estudos direcionados a composição fitoquimica da B. forficata apontam uma ampla composição que englobam flavanóides 
(Kaempferitrina, Kaempferol- 3,7-O- $\alpha$ - Diraminosídio, quercetina) e, terpenos (Isofitol, $\alpha$-humuleno, $\beta$-pineno, $\beta$ ocimeno, $\alpha$-pineno, $\beta$-cariofileno, biciclogermacreno) $(\text { Tabela } 1)^{8,16}$.

Tabela 1. Metabólitos secundários isolados da Bauhinia forficata

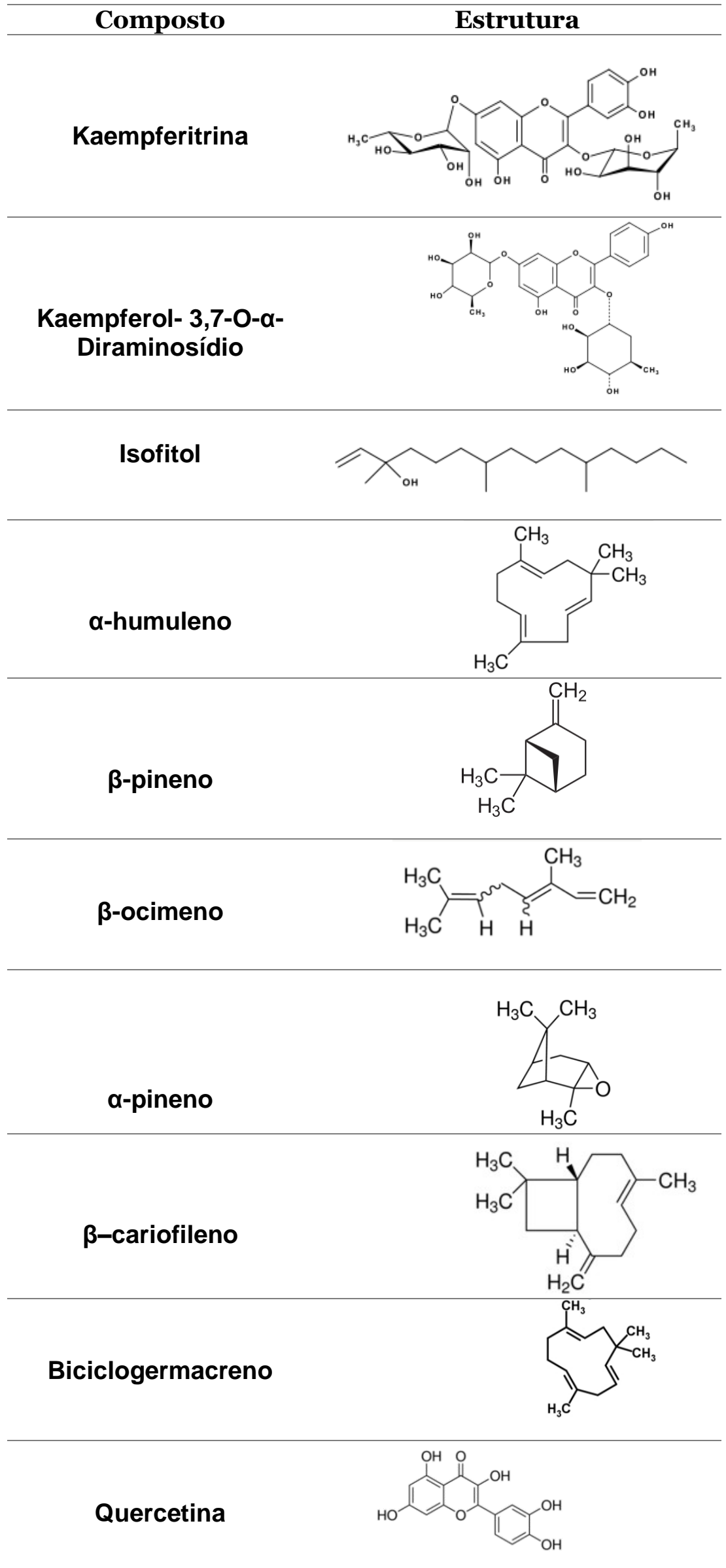

Fonte: Modificado de Menezes et al. ${ }^{8}$ 2007; Santos $^{16}, 2013$

- Propriedades farmacológicas da planta, com ênfase em hipoglicemiante

Por muito tempo a $B$. forficata vem sendo utilizada por parte da população como forma de controle da diabetes, através do uso do extrato aquoso de suas folhas e raízes, reforçando cada vez mais seu possível potencial terapêutico ${ }^{17}$.

Posteriormente, estudos realizados por Curcio et al. ${ }^{18}$ em camundongos normoglicêmicos e hiperglicêmicos, no qual se fez uso do extrato aquoso da pata-de-vaca, foi possível evidenciar o ganho e a recuperação de peso de camundongos diabéticos, embora não tenha sido eficaz no combate aos danos teciduais causados pela patologia.

Foram observados também resultados promissores com o uso dos flavonoides canferitrina e canferol presentes na composição química da planta ${ }^{15}$.

Uma das teorias bem aceitas a respeito do mecanismo de ação relacionado a redução da glicemia sanguínea pode ser atribuída a inibição da enzima responsável por catalisar o processo da digestão de açucares, podendo ainda serem relacionados a quercetina e ao canferol (kaempferol) pois ambos possuem estruturas que favorecem sua interação com a $\alpha$-glicosidade ${ }^{19}$.

Em relação ao emprego da $B$. forficata em formas farmacêuticas, um estudo científico aponta que o uso de extratos secos obtidos por spray-drying e granulação seca apresentam atividade hipoglicemiante a partir de concentrações de $200 \mathrm{mg} / \mathrm{kg}^{20}$.

\section{CONCLUSÃO}

Com base na metodologia utilizada e resultados encontrados concluiu-se que a $B$. forficata (pata-de-vaca) tem sido utilizada desde a antiguidade por diversos povos, devido sua ampla variedade de usos terapêuticos. Dentre estes, destaca-se o efeito hipoglicemiante do seu extrato já comprovado cientificamente, com o emprego em diferentes formas farmacêuticas, desde pós a granulados. Assim, a $B$. forficata mostra-se uma alternativa terapêutica promissora para o tratamento de diabetes mellitus além de outras patologias.

Embora a $B$. forficata se mostre bastante promissora para a área farmacêutica se faz necessário cada vez mais estudos associados a possíveis formas farmacêuticas que podem ser utilizadas, uma vez que o emprego de plantas medicinais na terapia de controle da diabetes seja uma forma de facilitar a adesão dos pacientes ao tratamento.

\section{REFERÊNCIAS}

1. Oliveira JEP, Vencio S (org). Diretrizes da sociedade brasileira de diabetes (2015-2016). São Paulo: A.C. Farmacêutica; 2016.

2. Salvi LC, Bersch B, Rempel C, Strohschoen AAG. Percepção de indivíduos com Diabetes Mellitus sobre a utilização de plantas medicinais hipoglicemiantes. Rev Contexto \& Saúde, 2016; 16(30):55-63.

3. Benini EB, Sartori MAB, Busch GC, Rempel C, Schultz G, Strohschoen AAG. Valorização da flora nativa quanto ao potencial fitoterápico. Destaques Acadêmicos. 2010; 2(3): 11-7.

4. Simões RC, Almeida SSMS. Estudo fitoquímico de Bauhinia forficata (Fabaceae). Biota Amazônia. 2015;5(1), 27-31.

5. Barbosa-Filho JM, Vasconcelos THC, Alencar AA, Batista LM, Oliveira RAG, Guedes DN et al. Plants and their active constituents from South, Central, and North America with hypoglycemic activity. Rev bras farmacogn. 2005; 15(4):392-413

6. Silva MIG, Gondim APS, Nunes IFS, Sousa FCF. Utilização de fitoterápicos nas unidades básicas de atenção à saúde da família no município de Maracanaú (CE). Rev bras farmacogn. 2006; 16(4):455-62.

7. Agra MF, França PF, Barbosa-Filho JM. Synopsis of the plants known as medicinal and poisonous in Northeast of Brazil. Rev bras farmacogn. 2007; 17(1):114-40. 
8. Menezes FS, Minto ABM, Ruela HS, Kuster RM, Sheridan H, Frankish N. Hypoglycemic activity of two Brazilian Bauhinia species: Bauhinia forficata $\mathrm{L}$. and Bauhinia monandra Kurz. Rev Bras Farmacogn. 2007; 17(1): 8-13.

9. Engel IC, Ferreira RA, Cechinel Filho V, Meyre da Silva C. Controle de qualidade de drogas vegetais a base de Bauhinia forficata Link (Fabaceae). Rev Bras Farmacogn. 2008; 18(2):258-64.

10. Mendes KDS, Silveira RCCP, Galvão CM. Revisão integrativa: método de pesquisa para incorporação de evidências na saúde e na enfermagem. Texto Contexto Enferm. 2008; 17(4):758-64.

11. Pereira ACS, Ribeiro GE, Souza LCR, Rufino LRA, Cabral ISR, Boriollo MFG et al. Atividade biológica do extrato hidroalcoólico de Bauhinia forficata Link sobre Herpetomonas samuelpessoai (Galvão.) Roitman. Rev Bras Pl Med. 2014;16(3):585-92.

12. Marques GS, Rolim LA, Alves LDS, Silva CCDAR, Soares LAL, Rolim-Neto PJ. Estado da arte de Bauhinia forficataLink (Fabaceae) como alternativa terapêutica para o tratamento do diabetes melittus. Rev Ciênc Farm Básica Apl. 2013; 34(3):313-20

13. Silva-López RE, Santos BC. Bauhinia forficata Link (Fabaceae). Fitos. 2015; 9(3):217-32.

14. Viana JS, Gonçalves EP, Andrade LA, Oliveira LSB, Silva EO. Crescimento de mudas de Bauhinia forficata Link em diferentes tamanhos de recipientes. Floresta, 2008; 38(4):636-71.

15. Trojan-Rodrigues M, Alves TL, Soares GL, Ritter MR. Plants used as antidiabetics in popular medicine in Rio Grande do Sul, southern Brazil. J Ethnopharmacol, 2012; 139(1):155-63.

16. Santos PMD. Estudos fitoquímicos de espécies do gênero Bauhinia (Fabaceae) da região Amazônica [dissertação]. Manaus: Universidade Federal do Amazonas; 2013.

17. Lima JFD. Estabelecimento da cultura de células de Bauhinia forficata Link como fonte de metabólitos bioativos [tese]. Ribeirão Preto: Faculdade de Ciências Farmacêutica de Ribeirão Preto, Universidade de São Paulo, Ribeirão Preto; 2009.

18. Curcio SA, Stefan LF, Randi BA, Dias MA, da Silva RE, Caldeira EJ. Hypoglycemic effects of an aqueous extract of Bauhinia forficata on the salivary glands of diabetic mice. Pak Pharm Sci. 2012; 25(3):493-9.

19. Ferreres F, Gil-Izquierdo A, Vinholes J, Silva ST, Valentão P, Andrade PB. Bauhinia forficata Link authenticity using flavonoids profile: Relation with their biological properties. Food Chem. 2012; 134(2):894-904.

20. Da Cunha A, Menon S, Menon R, Couto A, Bürger C, Biavatti M. Hypoglycemic activity of dried extracts of Bauhinia forficata Link. Phytomedicine. 2010; 17(1):37-41.

\section{CONFLITO DE INTERESSES}

Os autores declaram não haver conflitos de interesse.

\section{AUTOR PARA CORRESPONDÊNCIA}

\section{Maria Alana Neres de Pontes}

mariaalanapontes@gmail.com

Submetido em 25/08/2017

Aceito em 28/09/2017 